\title{
Biologia do Ácaro Predador Euseius alatus DeLeon (Acari: Phytoseiidae)
}

\author{
Paulo R. Reis ${ }^{1}$ e Everaldo B. Alves ${ }^{2}$ \\ ${ }^{1}$ EPAMIG/CRSM, Caixa postal 176, 37200-000, Lavras, MG. \\ ${ }^{2}$ Universidade Federal de Lavras, Caixa postal 37, 37200-000, Lavras, MG.
}

\author{
An. Soc. Entomol. Brasil 26(2): 359-363 (1997) \\ Biology of the Predaceous Mite Euseius alatus DeLeon \\ (Acari: Phytoseiidae)
}

\begin{abstract}
Euseius alatus DeLeon is a predaceous mite found on citrus plants (Citrus spp.) in Brazil, with a life cycle similar to the species of the same genus. Incubation period, larval, protonymphal and deutonymphal stages lasted ca. $24 \mathrm{~h}$ each, when mites were fed castor bean (Ricinus communis ) pollen and kept at $25 \pm 2{ }^{\circ} \mathrm{C}, 70 \pm 10 \% \mathrm{RH}$ and $14 \mathrm{~h}$ photophase. Oviposition period lasted 26.5 days, and the oviposition rate was 1.4 eggs/day. The intrinsic rate of increase $\left(\mathrm{r}_{\mathrm{m}}\right)$ was 0.217 ; population increased 1.2 times a day and double its population every 3.2 days. The population of E. alatus increase 21.4 times $\left(\mathrm{R}_{\mathrm{o}}\right)$ in the period of one generation $(\mathrm{T}=14.1$ days $)$.
\end{abstract}

KEY WORDS: Acari, life table, biological control, citrus, Citrus sinensis.

RESUMO - Euseius alatus DeLeon é um ácaro predador encontrado em plantas cítricas (Citrus spp.) no Brasil, que apresenta um ciclo de vida semelhante aos demais ácaros do mesmo gênero. O tempo de incubação e a duração das fases de larva, protoninfa e deutoninfa foi de aproximadamente $24 \mathrm{~h}$ cada, quando alimentado com pólen de mamoneira (Ricinus communis), a $25 \pm 2{ }^{\circ} \mathrm{C}, 70 \pm$ $10 \%$ UR e 14 h de fotofase. O período médio de oviposição foi de 26,5 dias, e a oviposição média foi de 1,4 ovos/dia. A capacidade inata de crescimento da população $\left(\mathrm{r}_{\mathrm{m}}\right)$ foi estimada em 0,217 , crescendo a população 1,2 vezes ao dia e dobrando a cada 3,2 dias. A população de E. alatus pode aumentar 21,4 vezes $\left(\mathrm{R}_{\mathrm{o}}\right)$ no tempo médio de uma geração $(\mathrm{T}=14,1$ dias $)$.

PALAVRAS-CHAVE: Acari, tabela de vida, controle biológico, citros, Citrus sinensis.

Euseius alatus DeLeon foi descrito por DeLeon (1966) sobre Cassia bicapsularis em Georgetown, nas Guianas Britânicas. As espécies de ácaros do gênero Euseius têm sido relatadas como predadoras eficientes de ácaros fitófagos de várias espécies e em diversos cultivos. Segundo Muma (1971), as espécies do gênero Euseius são polinífagas e alimentam-se facultativamente em grande número de insetos e ácaros. Esta característica pode ser interessante para a sobrevivência dos ácaros predadores na ausência de seu alimento 
preferido.

No Brasil, as espécies mais estudadas desse gênero têm sido Euseius citrifolius Denmark \& Muma (Moraes \& McMurtry 1981, Moreira 1993, Gravena et al. 1994) e Euseius concordis (Chant) (Moraes \& Lima 1983, Komatsu 1988). Quanto a E. alatus, há apenas relatos de sua ocorrência, principalmente em citros (Moraes \& McMurtry 1983, Delalibera et al. não publicado, Sato et al. 1994), cafeeiro (Coffea arabica) (Moraes \& McMurtry 1983, Pallini $\mathrm{F}^{\circ}$ et al.1992) na região Sudeste, e várias outras plantas no Nordeste (Moraes et al. 1993).

A presença deste ácaro em citros pode ser muito importante para o controle biológico dos ácaros-praga dessa cultura. Os resultados do presente trabalho contribuem para melhor conhecimento da biologia deste predador.

\section{Material e Métodos}

Dados da biologia de E. alatus foram obtidos no Laboratório de Acarologia do Centro Regional de Pesquisa do Sul de Minas, da Empresa de Pesquisa Agropecuária de Minas Gerais, em Lavras (MG), a $25 \pm 2$ ${ }^{\circ} \mathrm{C}, 70 \pm 10 \%$ de UR e $14 \mathrm{~h}$ de fotofase.

Os ácaros para estudo foram coletados em laranjeira 'Valência' (Citrus sinensis ), no pomar da Universidade Federal de Lavras, latitude $21^{\circ} 45^{\prime} \mathrm{S}$, longitude $45^{\circ} 00^{\prime} \mathrm{W}$ e altitude de $920 \mathrm{~m}$.

Todas as características biológicas do ácaro foram obtidas com pólen de mamoneira (Ricinus communis ) como alimento, e em arenas de $3 \mathrm{~cm}$ de diâmetro, confeccionadas com lâmina plástica flexível de cor preta, flutuando em água numa placa de Petri de 15 $\mathrm{cm}$ de diâmetro por $2 \mathrm{~cm}$ de profundidade, sem tampa. Em cada placa, foram colocadas oito arenas. As demais particularidades do método foram as mesmas descritas por Reis (1996). A água, além de servir de barreira, era utilizada também pelos ácaros para ingerir, o que é necessário quando recebem somente pólen como alimento (Muma 1971, Blommers et al. 1977, Sabelis 1981).
Criação Estoque. Foi feita com a mesma metodologia utilizada no estudo da biologia, exceto que os discos de lâmina plástica (arenas) foram maiores (6 cm de diâmetro), e colocados a flutuar em água, em bandejas plásticas $(32 \times 26,5 \times 5,5 \mathrm{~cm}$ de dimensões internas - 12 arenas por bandeja).

Período Embrionário. Os ovos, com 0 a 8 horas de idade, utilizados para estudo do tempo de incubação, foram obtidos nas arenas de criação estoque. Foram mantidos individualizados e observados diariamente às 8 e 16 h, para que fosse determinado o momento da eclosão.

Desenvolvimento Pós-Embrionário. Após a incubação os ácaros foram observados diariamente às 8 e $16 \mathrm{~h}$ para que fosse determinada a duração de cada estágio imaturo. Na fase adulta, os ácaros foram observados apenas uma vez ao dia, às $8 \mathrm{~h}$.

Tabela de Vida de Fertilidade. Foi confeccionada segundo metodologia de Andrewartha \& Birch (1954), citados por Silveira Neto et al. (1976) e de Lotka (1924) e Birch (1948), citados por Sabelis (1985). Para início do trabalho, os casais foram formados coletando-se deutoninfas que foram postas juntamente com machos nas arenas. As fêmeas foram facilmente identificadas pelo fato dos machos apresentarem o comportamento de segurar a deutoninfa pela parte posterior do idiossoma utilizando o primeiro par de pernas. Cada casal foi colocado em uma arena e observado a cada 24 horas, retirando-se os ovos postos no período e anotando-se o número de ácaros mortos. Os machos mortos eram substituídos por novos machos, provenientes da criação estoque, pois sabe-se que as fêmeas de fitoseídeos podem parar de colocar ovos na ausência de machos (Huffaker 1958, Chant 1959).

Foram calculadas a taxa líquida de reprodução $\left(\mathrm{R}_{\mathrm{o}}\right)$, duração média de uma geração $(T)$, capacidade inata de crescimento da população $\left(\mathrm{r}_{\mathrm{m}}\right)$ e a razão finita de aumento 
( $\lambda$ ). O valor de $r_{m}$ foi corrigido interativamente através da equação $\Sigma e^{-\mathrm{rm} \cdot \mathrm{x}} \cdot 1_{\mathrm{x}} \cdot \mathrm{m}_{\mathrm{x}}=1$ (Lotka 1924, Birch 1948, citados por Sabelis 1985). Com o valor corrigido de $\mathrm{r}_{\mathrm{m}}$, foi recalculada a duração média de uma geração $(\mathrm{T})$. O tempo necessário para que a população dobre, foi calculado pela relação $\ln 2 / \mathrm{r}_{\mathrm{m}}$ (Tanigoshi et al. 1975).

\section{Resultados e Discussão}

Período Embrionário. O tempo de incubação foi em média pouco superior a um dia, tanto para fêmeas como para machos (Tabela 1). Até um dia após a postura, 53,3\% das larvas eclodiram. O restante eclodiu no segundo dia. fêmeas (Tabela 1), o que resultou em um período médio de 4,5 dias de ovo a adulto, para ambos os sexos. Estes resultados estão próximos dos encontrados para E. citrifolius por Moraes \& McMurtry (1981) e Moreira (1993), e para E. concordis por Moraes \& Lima (1983) e Komatsu (1988). Na Tabela 2, estão relatados os períodos de pré-oviposição, oviposição e pós-oviposição, bem como a longevidade de adultos, machos e fêmeas, acasalados ou não. Tanto machos como fêmeas, quando acasalados, apresentaram menor longevidade do que quando não acasalados.

Foi constatado que E. alatus não se alimentou na fase de larva, fato já relatado

Tabela 1. Duração dos estágios imaturos de Euseius alatus (em dias), a $25 \pm 2{ }^{\circ} \mathrm{C}, 70 \pm$ $10 \%$ de UR e 14 h de fotofase, tendo como alimento pólen de mamoneira ( $\mathrm{n}=41$ fêmeas e 32 machos).

\begin{tabular}{llccc}
\hline \multirow{2}{*}{ Fases } & Sexo & \multicolumn{3}{c}{ Duração } \\
\cline { 3 - 5 } & & Mínima & Máxima & Média \pm EP \\
\hline \multirow{2}{*}{ Ovo } & Fêmea & 0,33 & 2,0 & $1,2 \pm 0,07$ \\
\multirow{2}{*}{ Larva } & Macho & 0,33 & 2,0 & $1,2 \pm 0,10$ \\
\multirow{2}{*}{ Protoninfa } & Fêmea & 0,33 & 2,7 & $1,2 \pm 0,07$ \\
\multirow{2}{*}{ Deutoninfa } & Macho & 0,33 & 2,3 & $1,1 \pm 0,06$ \\
& Fêmea & 0,33 & 2,7 & $1,1 \pm 0,05$ \\
\multirow{2}{*}{ Ovo-adulto } & Fêmea & 0,33 & 2,0 & $1,1 \pm 0,05$ \\
& Macho & 0,33 & 2,0 & $1,0 \pm 0,04$ \\
& Fêmea & 0,33 & 1,7 & $1,1 \pm 0,04$ \\
& Macho & 1,32 & 9,4 & $4,5 \pm 0,23$ \\
\hline
\end{tabular}

Desenvolvimento Pós-Embrionário. Após a fase de ovo, E. alatus passou pelas fases de larva, protoninfa e deutoninfa, como ocorre com as demais espécies de ácaros da família Phytoseiidae, antes de atingir a fase adulta. As fases de larva, protoninfa e deutoninfa apresentaram, cada uma, duração média superior a um dia, tanto para machos como para para outras espécies de fitoseídeos (Chant 1959).

Oviposição. E. alatus apresentou um período médio de oviposição de 26,5 dias, cada fêmea pondo cerca de $38 \pm 4,36$ ovos durante esse período ( $\mathrm{n}=12$ fêmeas), com uma média de $1,4 \pm 0,13$ ovos/fêmea /dia. 
Tabela 2. Duração de diversas fases do ciclo evolutivo de Euseius alatus (em dias), a $25 \pm$ $2{ }^{\circ} \mathrm{C}, 70 \pm 10 \%$ de UR e $14 \mathrm{~h}$ de fotofase, tendo como alimento pólen de mamoneira.

\begin{tabular}{lrrrr}
\hline & & \multicolumn{3}{c}{ Duração } \\
\cline { 3 - 5 } Fases & $\mathrm{n}$ & Mínima & Máxima & Média \pm EP \\
\hline Pré-oviposição & 11 & 1,3 & 2,7 & $1,8 \pm 0,13$ \\
Oviposição & 11 & 20,0 & 34,0 & $26,5 \pm 1,41$ \\
Pós-oviposição & 7 & 3,0 & 7,0 & $5,0 \pm 0,49$ \\
Adulto: & & & & \\
Fêmea acasalada & 11 & 15,0 & 35,0 & $30,2 \pm 1,69$ \\
Fêmea virgem & 39 & 9,0 & 84,0 & $56,1 \pm 2,35$ \\
Macho acasalado & 23 & 5,0 & 32,0 & $15,1 \pm 1,53$ \\
Macho virgem & 25 & 4,0 & 27,0 & $19,6 \pm 1,88$ \\
\hline
\end{tabular}

Razão Sexual. Foi encontrada uma proporção de 1,3 fêmeas para 1 macho, resultado semelhante ao relatado por Moreira (1993) para E. citrifolius.

Tabela de Vida de Fertilidade. Foi estimado um valor de $\mathrm{r}_{\mathrm{m}}=0,217$ fêmeas /fêmea /dia, o que indicou que a população de E. alatus cresceu 1,2 vezes ao dia (razão finita de aumento, $\lambda=1,2$ ) e dobrou a cada 3,2 dias. A taxa líquida de reprodução $\left(\mathrm{R}_{\mathrm{o}}\right)$ apresentou um valor de 21,4, o que representa o número médio de fêmeas nascidas no tempo de vida de cada fêmea, ou seja, o número de vezes que a população aumenta a cada geração. A duração média de uma geração (T) foi de 14,1 dias, o que significa que a população de $E$. alatus é estimada em aumentar 21,4 vezes no tempo médio de uma geração.

Os resultados mostraram que E. alatus apresentou características biológicas semelhantes a outros ácaros criados em laboratório e já em uso no controle biológico, sendo portanto um ácaro que tem potencial para uso semelhante, com a vantagem de poder ser criado com pólen de mamoneira como alimento.

\section{Agradecimentos}

À Fundação de Amparo à Pesquisa do
Estado de Minas Gerais (FAPEMIG) pelo auxílio financeiro para a execução da pesquisa e concessão de bolsa de Iniciação Científica a Everaldo B. Alves. Ao Dr. Gilberto J. de Moraes, da ESALQ /USP, pela confirmação da identificação da espécie do ácaro.

\section{Literatura Citada}

Blommers, L., P. Lobbes, P. Vink \& F. Wegdam. 1977. Studies on the response of Amblyseius bibens (Acarina: Phytoseiidae) to conditions of prey scarcity. Entomophaga 22: 247-258.

Chant, D.A. 1959. Phytoseiid mites (Acarina: Phytoseiidae). Part I - Bionomics of seven species in southeastern England. Part II A taxonomic review of the family Phytoseiidae, with descriptions of thirty eight new species. Can. Entomol. 91: 1166.

DeLeon, D. 1966. Phytoseiidae of British Guyana, with keys to species (Acari: Mesostigmata), In: Martinus Nijhoff (ed.). Studies on the fauna of Suriname and other Guyanas. The Hague, v.8, p.81-102.

Gravena, S., I. Benetoli, P.H.R. Moreira \& 
P.T. Yamamoto. 1994. Euseius citrifolius Denmark \& Muma predation on citrus leprosis mite Brevipalpus phoenicis (Geijskes) (Acari: Phytoseiidae: Tenuipalpidae). An. Soc. Entomol. Brasil 23: 209-218.

Huffaker, C.B. 1958. Experimental studies on predation: dispersion factors and predator-prey oscillations. Hilgardia 27: 343-383.

Komatsu, S.S. 1988. Aspectos bioetológicos de Euseius concordis (Chant, 1959) (Acari: Phytoseiidae) e seletividade dos acaricidas convencionais nos citros. Tese de mestrado, ESALQ/USP, Piracicaba, $117 \mathrm{p}$.

Moraes, G.J. de \& H.C. Lima. 1983. Biology of Euseius concordis (Chant) (Acarina: Phytoseiidae) a predator of the tomato russet mite. Acarologia 24: 251255.

Moraes, G.J. de \& J.A. McMurtry. 1981. Biology of Amblyseius citrifolius (Denmark \& Muma) (Acari: Phytoseiidae). Hilgardia 49: 1-29.

Moraes, G.J. de \& J.A. McMurtry. 1983. Phytoseiid mites (Acarina) of northeastern Brazil with descriptions of four new species. Internat. J. Acarol. 9: 131148.

Moraes, G.J. de, J.A. de Alencar, J.L.S. de Lima, J.S. Yaninek \& I. Delalibera Jr. 1993. Alternative plant habits for common phytoseiid predators of the cassava green mite (Acari: Phytoseiidade, Tetranychidae) in Northeast Brazil. Exp. \& Appl. Acarol. 17: 77-90.

Moreira, P.H.R. 1993. Ocorrência, dinâmica populacional de ácaros predadores em citros e biologia de Euseius citrifolius (Acari: Phytoseiidae). Tese de mestrado, FCAV/UNESP, Jaboticabal, 110p.
Muma, M.H. 1971. Food habits of Phytoseiidae (Acarina: Mesostigmata) including common species on Florida citrus. Fla. Entomol. 54: 21-34.

Pallini $\mathrm{F}^{\circ}$, A., G.J. de Moraes \& V.H.P. Bueno. 1992. Ácaros associados ao cafeeiro (Coffea arabica L.) no Sul de Minas Gerais. Ciênc. Prát. 16: 303-307.

Reis, P.R. 1996. Aspectos biológicos e seletividade de agroquímicos a Iphiseiodes zuluagai Denmark \& Muma (Acari: Phytoseiidae). Tese de doutorado, ESALQ/USP, Piracicaba, 154p.

Sabelis, M.W. 1981. Biological control of two-spotted spider mites using phytoseiid predators. Part I: Modelling the predatorprey interation at the individual level. Wageningen, Cent. for Agric. Publ. Document., 242p.

Sabelis, M.W. 1985. Capacity for population increase. p.35-41. In: W. Helle, \& M.W. Sabelis (eds.). Spider mites; their biology, natural enemies and control. Amsterdam, Elsevier, v.1B, 428p.

Sato, M.E., A. Raga, L.C. Cerávolo, A.C. Rossi \& M.R. Potenza. 1994. Ácaros predadores em pomar cítrico de Presidente Prudente, Estado de São Paulo. An. Soc. Entomol. Brasil 23: 435 441.

Silveira Neto, S., O. Nakano, D. Barbin \& N.A. Villa Nova. 1976. Manual de ecologia dos insetos. São Paulo, Ceres, $419 \mathrm{p}$.

Tanigoshi, L.K., S.C. Hoyt, R.W. Browne \& J.A. Logan. 1975. Influence of temperature on population increase of Metaseiulus occidentalis (Acarina: Phytoseiidae). Ann. Entomol. Soc. Am. 68: 979-986.

Recebido em 27/05/96. Aceito em 25/07/97. 